\title{
Blue Light Stimulates Cognitive Brain Activity in Visually Blind Individuals
}

\author{
Gilles Vandewalle ${ }^{1,2 * * * *}$, Olivier Collignon ${ }^{3,4 *, \dagger}$, Joseph T. Hull $^{5,6}$, \\ Véronique Daneault $^{1,2}$, Geneviève Albouy ${ }^{1}$, Franco Lepore ${ }^{3}$, \\ Christophe Phillips ${ }^{7}$, Julien Doyon ${ }^{1}$, Charles A. Czeisler ${ }^{5,6}$, \\ Marie Dumont ${ }^{2}$, Steven W. Lockley ${ }^{5,6 \dagger \dagger}$, and Julie Carrier ${ }^{1,2 \dagger \dagger}$
}

\begin{abstract}
Light regulates multiple non-image-forming (or nonvisual) circadian, neuroendocrine, and neurobehavioral functions, via outputs from intrinsically photosensitive retinal ganglion cells (ipRGCs). Exposure to light directly enhances alertness and performance, so light is an important regulator of wakefulness and cognition. The roles of rods, cones, and ipRGCs in the impact of light on cognitive brain functions remain unclear, however. A small percentage of blind individuals retain non-image-forming photoreception and offer a unique opportunity to investigate light impacts in the absence of conscious vision, presumably through ipRGCs. Here, we show that three such patients were able to choose nonrandomly about the presence of light despite their complete lack of sight. Furthermore, $2 \mathrm{sec}$ of blue light modified EEG activity when administered simultaneously to auditory stimulations. fMRI further showed that, during an
\end{abstract}

\section{INTRODUCTION}

Light is essential for the regulation of numerous circadian, neuroendocrine, and neurobehavioral functions, sometimes termed nonvisual or non-image-forming responses, via outputs from intrinsically photosensitive retinal ganglion cells (ipRGCs; Schmidt, Chen, \& Hattar, 2011; Hatori \& Panda, 2010). These ipRGCs represent a recently discovered novel class of retinal photoreceptors (in addition to rods and cones), which express the photopigment melanopsin, are maximally sensitive to short-wavelength blue light $(\sim 480 \mathrm{~nm})$, and project broadly throughout

\footnotetext{
${ }^{1}$ University of Montréal Geriatric Institute, Québec, Canada, ${ }^{2}$ Hôpital du Sacré-Cour de Montréal, Québec, Canada, ${ }^{3}$ Université de Montréal, Québec, Canada, ${ }^{4}$ Centre de Recherches CHU Sainte-Justine, Montréal, Québec, Canada, ${ }^{5}$ Brigham and Women's Hospital, Boston, MA, ${ }^{6}$ Harvard Medical School, Boston, MA, ${ }^{7}$ University of Liège, Belgium

*These authors contributed equally to this work.

**Present address: Cyclotron Research Centre, University of Liège, Belgium.

${ }^{\dagger}$ Present address: Centre for Mind/Brain Science, University of Trento, Italy.

these authors are joint senior authors on this work.
}

auditory working memory task, less than a minute of blue light triggered the recruitment of supplemental prefrontal and thalamic brain regions involved in alertness and cognition regulation as well as key areas of the default mode network. These results, which have to be considered as a proof of concept, show that non-image-forming photoreception triggers some awareness for light and can have a more rapid impact on human cognition than previously understood, if brain processing is actively engaged. Furthermore, light stimulates higher cognitive brain activity, independently of vision, and engages supplemental brain areas to perform an ongoing cognitive process. To our knowledge, our results constitute the first indication that ipRGC signaling may rapidly affect fundamental cerebral organization, so that it could potentially participate to the regulation of numerous aspects of human brain function.

the brain. Importantly, light exposure can directly enhance alertness and performance during multiple cognitive tasks, with a greater efficiency for short wavelength light, so that light is an essential regulator of wakefulness and cognition (Chellappa, Gordijn, \& Cajochen, 2011; Lockley et al., 2006). The brain mechanisms involved in the stimulant effect of light on cognitive function are only starting to be elucidated, however (Vandewalle, Maquet, \& Dijk, 2009).

Human neuroimaging has demonstrated that ocular light exposure acutely modulates attentional, executive, and emotional brain responses to auditory tasks (Vandewalle et al., 2006, 2009, 2010, 2011; Vandewalle, Gais, et al., 2007; Vandewalle, Schmidt, et al., 2007; Perrin et al., 2004). These studies identified the hypothalamus, the thalamus pulvinar (PULV), and the brainstem locus coeruleus as likely subcortical interfaces mediating the nonimage-forming impact of light on the cortical areas involved in the ongoing cognitive process, particularly in prefrontal and parietal cortices. IpRGCs are likely to be the primary photoreceptors mediating these effects as, compared with other wavelengths, blue light is more effective in inducing sustained modulations of brain responses. A direct demonstration of the implications of a non-image-forming photoreception system in the light-induced modification 
of cognitive brain activity is still lacking, however, because prior neuroimaging data were acquired in fully sighted participants (Allen, Brown, \& Lucas, 2011; Lall et al., 2010; Dkhissi-Benyahya, Gronfier, De Vanssay, Flamant, \& Cooper, 2007).

Nearly two decades ago, it was discovered that retinal light exposure could suppress melatonin and entrain circadian rhythms in some blind people, despite a complete absence of conscious light perception (Czeisler et al., 1995). Later research confirmed this finding and established that light could also induce circadian phase resetting and slow-onset pupil constriction in this small percentage of totally visually blind individuals with outer retina degeneration but presumably with an intact ganglion cell layer (Zaidi et al., 2007; Klerman et al., 2002). These responses are similar to the circadian and pupil responses to light observed in rodents lacking functional rods and cones, which are mediated through ipRGC photoreception (Hattar et al., 2003; Panda et al., 2003; Lucas, Freedman, Muñoz, Garcia-Fernández, \& Foster, 1999). In addition, in one of these individuals, prolonged exposure to light improved subjective and objective EEG correlates of alertness as well as performance during a simple auditory psychomotor vigilance task (PVT; Zaidi et al., 2007). This non-image-forming impact of light is likely to arise from ipRGCs because (1) these cells are known to be preserved in individuals with outer retinal degeneration (Hannibal et al., 2004), (2) ophthalmological examination confirmed atrophy of the retinal pigment epithelium and found no detectable functional responses from rods and cones (Zaidi et al., 2007), (3) light effects were more pronounced using 460- to 480-nm monochromatic light as compared with other wavelengths (Zaidi et al., 2007), and (4) recent data show that the dynamics of pupillary constriction in a blind human with outer retinal degeneration was compatible with the exclusive involvement of ipRGCs (Gooley et al., 2012). Blind individuals with preserved non-image-forming photoreception might also exhibit some nonconscious awareness of light. In a previous report, despite complete visual blindness, one participant was able to choose successfully when a light exposure was presented in a twoalternative forced-choice task (2AFC) but only when 480-nm blue monochromatic light, and not other wavelengths, were administered (Zaidi et al., 2007). These rare individuals - only nine have been identified to date worldwide-therefore offer a unique opportunity to investigate the selective impact of light on cognitive brain functions in the absence of conscious vision, presumably solely via ipRGCs' intrinsic light sensitivity.

In this study, we aimed to confirm and extend the finding that nonconscious awareness of light is apparent in visually blind participants who retain non-imaging-forming responses to light. In addition, we used a traditional EEG protocol, adapted from the multisensory integration literature, to investigate whether brief exposure (up to $2 \mathrm{sec}$ ) to high-intensity blue light could modify EEG activity in these participants while they performed an auditory cognitive task. Finally, we used fMRI to test whether exposure to high-intensity blue light for less than a minute modulated cognitive brain responses to an auditory task and to identify neural correlates of this light-induced modulation.

\section{METHODS}

\section{Participants}

Three totally visually blind individuals, with complete loss of sight and no conscious light perception, participated in this study (60-67 years old, one woman; see Table 1 for detailed characteristics). They provided written informed consent, and all experiments were approved by the Comité mixte d'éthique de la recherche du Regroupement Neuroimagerie/Québec. All three had previously completed studies that established that they retained a light-induced melatonin suppression response despite the absence of conscious vision (published for two of them: Zaidi et al., 2007; Klerman et al., 2002). Participant 1 had pupil muscle damages during lens removal surgery for cataract problems and did not respond to a standard pen-light examination. Participant 2 exhibited pupil constriction if the pen-light exposure was continued for up to $10 \mathrm{sec}$. Participant 3 had no clearly distinguishable pupil. In previous visits to Boston, a fundoscopic examination in Participants 1 and 2 confirmed atrophy of the retinal pigment epithelium, with thinning of retinal vessels and bone spicule pigmentation. These findings were confirmed on several occasions by different ophthalmologists who examined the patient independently. Visual acuity tests previously performed in Boston indicated no light perception in either eye of the three participants. Standard visually evoked EEG potential (VEP) procedures were also previously administered to Participants 1 and 2, and a standard electroretinogram procedure was previously administered to Participant 3. No classical visual responses were detected in any tests. Finally, questionnaire scores indicated that Participants 2 and 3 reported, respectively, relatively poor sleep quality and high propensity to fall asleep during the day (Table 1).

All three participants had been declared totally blind for at least 10 years, but the cause and duration of sight loss differed (Table 1). Participants maintained a regular sleep schedule for 7 days before travelling to Montréal (verified using sleep logs). They remained on their home time zone for their entire stay in Montréal and were allowed to go outside when not performing an experiment. No pupil dilator was administered for any of the experiments described below.

\section{EEG Protocol}

On two consecutive days, participants arrived in the laboratory $6.5 \mathrm{~h}$ after wake time and were blindfolded for $1 \mathrm{~h}$ 
Table 1. Participants Characteristics

\begin{tabular}{|c|c|c|c|}
\hline & Participant 1 & Participant 2 & Participant 3 \\
\hline Age & 67 & 60 & 66 \\
\hline Sex & Female & Male & Male \\
\hline Body mass index & 27.3 & 30.0 & 27.4 \\
\hline Cause of blindness & Retinitis pigmentosa & Retinitis pigmentosa & Retinopathy of prematurity \\
\hline Years of total blindness & 10 & 25 & 66 \\
\hline Chronotype & $\begin{array}{l}\text { 62, moderate morning } \\
\text { type }\end{array}$ & $\begin{array}{l}\text { 72, moderate morning } \\
\text { type }\end{array}$ & $\begin{array}{l}68, \text { moderate morning } \\
\text { type }\end{array}$ \\
\hline $\begin{array}{l}\text { Anxiety (from } 0=\text { lowest to } \\
\quad 63=\text { highest })\end{array}$ & 5 & 0 & 2 \\
\hline \multicolumn{4}{|l|}{ Mood (from $0=$ best to $63=$ worst) } \\
\hline $\begin{array}{l}\text { Sleep disturbances (from } 0=\text { lowest } \\
\text { to } 21=\text { highest) }\end{array}$ & 3 & 10 & 4 \\
\hline $\begin{array}{l}\text { Daytime propensity to } \\
\text { fall asleep }\end{array}$ & 1 & 4 & 16 \\
\hline Laterality & Right handed & Right handed & Right handed \\
\hline Time zone difference with Montréal & $-1 \mathrm{~h}$ & $0 \mathrm{~h}$ & $-3 \mathrm{~h}$ \\
\hline Cigarette consumption & No & No & No \\
\hline Medication & None & None & $\begin{array}{l}\text { Low dose of blood pressure } \\
\text { medication }\end{array}$ \\
\hline Years of education & 17 & 12 & 12 \\
\hline Alcohol consumption (units/week) & $<1$ & $<1$ & 0 \\
\hline Caffeine consumption (cups/day) & 2 & 5 & 2 \\
\hline $\begin{array}{l}\text { Last confirmation of light-induced } \\
\text { melatonin suppression }\end{array}$ & Yes, in 2008 & Yes, in 2006 & Yes, in 2002 \\
\hline $\begin{array}{l}\text { Previous standard ERG examination } \\
\quad \text { (no detectable signal) }\end{array}$ & No & No & Yes, in 1994 \\
\hline $\begin{array}{l}\text { Previous standard VEP examination } \\
\text { (no detectable signal) }\end{array}$ & Yes, in 2008 & Yes, in 2006 & No \\
\hline $\begin{array}{l}\text { Pupil response to prolonged } \\
\text { light exposure }(>5 \mathrm{sec})\end{array}$ & No & Yes & No \\
\hline Participated in a published study & No & Yes (Zaidi et al., 2007) & Yes (Klerman et al., 2002) \\
\hline
\end{tabular}

Chronotype was assessed by the Horne-Östberg Questionnaire (Horne \& Ostberg, 1976), anxiety level was measured on the 21-item Beck Anxiety Inventory (Beck, Epstein, Brown, \& Steer, 1988), mood was assessed using the 21-item Beck Depression Inventory II (Steer, Ball, Ranieri, \& Beck, 1997); sleep disturbance was determined by the Pittsburgh Sleep Quality Index Questionnaire (Buysse, Reynolds, Monk, Berman, \& Kupfer, 1989), and daytime propensity to fall asleep during daytime nonstimulating situations was assessed by the Epworth Sleepiness Scale (Johns, 1991). ERG = electroretinogram.

before the first recording was initiated. All recordings were conducted in a dark and sound-attenuated Faraday room while participants sat with their head on a chin rest and with their eyes $6 \mathrm{~cm}$ away from the center of a $21 \times$ $11 \mathrm{~cm}$ diffusion glass, including ultraviolet and infrared filters, behind which a 48 blue light-emitting diode (LED) array was placed (peak $=465 \mathrm{~nm}, \mathrm{FWHM}=27 \mathrm{~nm}$; spectrum assessed with Lightspex [GretagMacbeth, New Windsor, NY]; see Supplementary Figure S4).
Irradiance at eye level was high and set at $414 \mu \mathrm{W} / \mathrm{cm}^{2} / \mathrm{sec}$ (PM100D, Thorlabs, Newton, NJ), which corresponds to $9.7 \times 10^{14}$ photons $/ \mathrm{cm}^{2} / \mathrm{sec}$. The light device produced no perceptible sounds or temperature change. Throughout the EEG protocol, participants' "gaze" was monitored with an infrared camera to ensure that their eyes remained open. Presentation software (Neurobehavioral Systems, Inc., Albany, CA) was used to produce auditory stimuli, control the LED array, and record keyboard responses. Auditory 
stimuli were transmitted to the participant via headphones (EarTone3A, Etymotic Research, Elk Grove Village, IL). Volume was set to an individual comfortable level before the task was initiated.

\section{$2 A F C$}

At the start of the EEG protocol and after being blindfolded for at least $1 \mathrm{hr}$, participants were asked to report whether the high-irradiance blue LED array was on during the first or second half of a 4-, 10-, or 20-sec interval in a $2 \mathrm{AFC}$. Each trial started with the auditory instruction "start" and ended with the auditory instruction "end." A high-pitched sound (500 msec, $1000 \mathrm{~Hz}$ ) indicated the middle of the trial. The participant gave his or her response at the end of each trial both orally and by pressing a key. Blue light was pseudorandomly turned on in the first or second half of the trial. Each trial duration was tested in a separate session, which included 40 trials delivered to both eyes simultaneously. The 4 - and 10-sec trial durations were tested on the same day, whereas the 20 -sec trial duration was acquired on the preceding or following day. Cumulative binomial statistics on discrimination were carried out to determine whether responses were significantly different from random choices.

\section{Visually Evoked Potential}

Participants were instructed to keep their eyes wide open in front of the LED array while blinking as little as possible. Given the small sample size and their visual blindness, the number of trials was high (800) so that slight EEG-evoked responses could be detected. Flashes of blue light (duration $=500 \mathrm{msec}$, ISI $=1600-1800 \mathrm{msec}$ ) were delivered to the participant in four blocks of 200 stimuli (two blocks per experimental day).

\section{Bimodal PVT}

Visual stimuli consisted of 500-msec and 2-sec exposures to blue light. Auditory stimuli consisted of 150-msec pink noise bursts ( $90 \%$ of normalized peak value, plateau time $=90 \mathrm{msec}$, rise $/$ fall time $=5 \mathrm{msec}$ ). For bimodal stimuli, auditory stimuli were administered for the last 150 msec of the visual stimuli (i.e., 350 or $1850 \mathrm{msec}$ after light onset). Participants were asked to respond as fast as possible to each sound by pressing a keyboard with their right hand. Given the limited number of participants, a high number of trials were recorded. Participants completed five blocks of each conditions (500 msec and $2 \mathrm{sec}$ ), and each block included 300 trials equally divided between each stimuli type (100 sound alone, 100 light alone, 100 bimodal) for 500 trials per stimulus type. ISI was randomly ranged from 1200 to $2600 \mathrm{msec}$ (mean = $1900 \mathrm{msec}$ ) in the 500-msec condition and from 1200 to $1600 \mathrm{msec}($ mean $=1400 \mathrm{msec})$ in the 2 -sec condition.
Participants 2 and 3 started with the 500-msec condition, whereas Participant 1 started with the 2-sec condition.

\section{EEG Recordings and Analyses}

During the VEP and bimodal PVT protocols, EEG was recorded from $40 \mathrm{Ag}-\mathrm{AgCl}$ electrode caps (Neurosoft, Inc., Sterling, VA), placed according to the extended international 10-20 system, including a ground electrode. All electrodes were referred to both mastoids, and impedance was maintained at $<5 \mathrm{k} \Omega$. EEG and EOG were digitized at $1000 \mathrm{~Hz}$, high-pass filtered at $0.1 \mathrm{~Hz}$, low-pass filtered at $100 \mathrm{~Hz}$, and averaged offline.

Trials with artifacts at electrode sites of interest (Cz and $\mathrm{Oz}$ ) or eye blinks (vertical eye movements $>100 \mu \mathrm{V}$ ) were manually excluded. EEG time series of $600 \mathrm{msec}$, with 100-msec preauditory stimulus, were edited off-line using BrainVision Analyzer (Brain products, Gilching, Germany) following three steps: (1) data filtering (0.1$35 \mathrm{~Hz}$ ), (2) data epoching, and (3) baseline corrections. For trials with light stimuli alone of the bimodal PVT protocol, the last $150 \mathrm{msec}$ of the light pulse were considered as postauditory stimulus for comparison with trial with auditory stimulations (i.e., as if a sound had been produced). Edited EEG time series of each trial for each participant and for each condition were exported to Matlab 7.1 (MathWorks, Inc., Natick, MA) for analyses. For the VEP recording, trials were averaged and displayed in Supplementary Figure S1. Because no responses could be isolated, no statistical analyses were performed. For the bimodal PVT, as classically performed in EEG experiments investigating multisensory integration (Molholm et al., 2002; Giard \& Peronnet, 1999), ERPs from the auditory-alone and visual-alone conditions were summed for statistical comparison with the ERP response to the simultaneous audiovisual condition.

Given the limited sample size, conservative singlesubject analyses was undertaken. We used a nonparametric Monte Carlo permutation approach to find the time points, in each individual separately, with significant differences between the ERPs obtained in the bimodal condition and the sum of the ERPs obtained in the two unimodal conditions. For each time point, bimodal trials and the sum of the two unimodal trials were grouped in a single set, which was randomly partitioned 500 times between the two conditions. $t$ Statistics were computed for each partition to construct a histogram of $t$ statistic distribution. We finally tested for each time point whether the proportion of $t$ test of our permutations was above the $t$ test of our original conditions. This proportion is the Monte Carlo significance probability, which constitutes the $p$ value. To further control for false positives, we only considered significant differences present for 10 consecutive time points ( $\sim 10 \mathrm{msec}$ at a sampling rate of $1024 \mathrm{~Hz}$ ), because the likelihood of getting 10 false positives in a row is considerably low, even if these time points are nonindependent (Giard \& Peronnet, 1999). 


\section{fMRI Protocol}

Participants arrived at the laboratory $2.5 \mathrm{~h}$ before habitual sleep time. A structural image of the brain was acquired, and participants were familiarized with the MR setting. Participants were then blindfolded for $2 \mathrm{~h}$ before the fMRI run, which started $30 \mathrm{~min}$ after habitual sleep onset time. Light was produced by a quartz halogen white light source (PL950, Dolan-Jenner Industries, Boxborough, $\mathrm{MA}$ ), filtered by narrow interference band-pass filter (Edmund Optic, Barrington, NJ) to produce blue monochromatic light (peak $=480 \mathrm{~nm}, \mathrm{FWHM}=13 \mathrm{~nm}$; spectrum assessed with Lightspex; Supplementary Figure S4). Light was transmitted by a metal-free optical fiber diffusers (glasses frames mounted with $7 \times 9 \mathrm{~cm}$ uniform diffusing glass placed $2 \mathrm{~cm}$ away from the eye). Blue light irradiance at eye level was set at $81 \mu \mathrm{W} / \mathrm{cm}^{2}(1.95 \times$ $\left.10^{14} \mathrm{photons} / \mathrm{cm}^{2} / \mathrm{sec}\right)$. The light device produced no perceptible sounds or temperature change.

\section{2-Back Task}

Stimuli consisted of nine English monosyllabic consonants (duration $=500 \mathrm{msec}$, ISI $=2000 \mathrm{msec}$ ), produced using COGENT 2000 (www.vislab.ucl.ac.uk/cogent.php), implemented in MATLAB, and transmitted to the participants using MR CONTROL amplifier and headphones (MR Confon, Magdeburg, Germany). Data aquisition was preceded by a short session during which volunteers set volume level. For each auditory stimulus, volunteers were requested to state whether it was identical to the stimulus presented two stimuli earlier by pressing buttons with their right hand on an MR-compatible keypad. Series of stimuli were constructed with $\sim 30 \%$ hits so that difficulty was similar in all blocks, were presented only once, and were randomly assigned to a task block. Participants were trained to the task for $30 \mathrm{~min}$ in the afternoon preceding fMRI acquisition, and performance was $>88 \%$ in all three participants (supplementary results; Supplementary Figure S3A, top).

Participants performed 20 task blocks, which lasted $55 \mathrm{sec}$ and contained 22 auditory stimuli. Participants performed the task alternatively in complete darkness $(<0.01$ lux) or while exposed to blue light, in which case, the light was turned on and off at the beginning and end of a task block (and therefore, exposure to light also lasted $55 \mathrm{sec}$ ). Ten blocks included blue light, and 10 blocks included darkness. Blocks were separated by 13- to 22-sec episodes of rest in darkness $(<0.01$ lux, mean duration $=$ $17.6 \mathrm{sec}$ ). The first block for Participants 1 and 2 included blue light, whereas for Participant 3, the first block was conducted in darkness.

\section{Technical Issues}

For technical reasons, the initial fMRI data obtained in Participant 3 could not be used. Therefore, the follow- ing evening, this participant performed the identical fMRI protocol, with the exception that he was allowed to sleep later than usual the night preceding acquisitions (equal sleep duration but bedtime and waketime were delayed by $1.5 \mathrm{hr}$ ) to prevent sleep deprivation from the previous testing night.

\section{fMRI Data Acquisition}

Functional MRI time series were acquired using a 3-T MR scanner (TIM-TRIO, Siemens, Erlangen, Germany). Multislice $\mathrm{T} 2 *$-weighted fMRI images were obtained with a gradient-echo-planar sequence using axial slice orientation (32 slices, voxel size $=3.4 \times 3.4 \times 3 \mathrm{~mm}^{3}$ with $30 \%$ gap, matrix size $=64 \times 64 \times 32$, repetition time $=$ $2000 \mathrm{msec}$, echo time $=30 \mathrm{msec}$, flip angle $=90^{\circ}$ ). Structural brain images consisted of a T1-weighted 3-D magnetization prepared rapid gradient echo (repetition time $=7.92 \mathrm{msec}$, echo time $=2.4 \mathrm{msec}$, time of inversion $=$ $910 \mathrm{msec}$, flip angle $=15^{\circ}$, field of view $=256 \times 224 \mathrm{~mm}^{2}$, matrix size $=256 \times 224$, voxel size $=1 \times 1 \times 1 \mathrm{~mm}^{3}$ ).

\section{fMRI Data Analyses}

Data were analyzed using SPM8 (www.fil.ion.ucl.ac.uk/ spm). They were realigned, coregistered, and spatially normalized using the "new_segment" and "dartel" toolboxes of SPM8 (which includes smoothing). Because of the limited sample size, random effects analyses accounting for intersubject variability were not carried out, and the analysis consisted in a single fixed effects step including all three participants. This approach guarantees that any significant differences obtained in our analyses are valid within our sample of three subjects but implies that it cannot be generalized yet to the entire population of totally blind individuals with preserved non-imageforming photoreception. Changes in brain responses were estimated using a general linear model, in which task blocks were modeled using boxcar functions, convolved with a canonical hemodynamic response function and its first and second derivatives. Light onsets were modeled using stick function ("event"), convolved with a canonical hemodynamic response function and its first and second derivatives. A parametric modulation was added to each regressor to track any linear change of the amplitude of brain responses across time. Regressors were modeled separately in task blocks performed under blue light exposure and in complete darkness. Movement parameters derived from functional volumes realignment were considered as covariates of no interest. High-pass filtering was implemented in the matrix design using a cutoff period of $256 \mathrm{sec}$ to remove low-frequency drifts from the time series. Serial correlations in the fMRI signal were estimated using autoregressive (order 1) plus white noise model and restricted maximum likelihood algorithm. The contrasts of interest compared task blocks performed under blue light and in 
darkness. Statistical inferences on the $t$ statistics maps resulting from contrasts of interest were performed at a threshold of $p=.05$ after conservative correction for multiple comparisons computed on the entire brain volume (family-wise error method).

\section{RESULTS}

We conducted classical visually evoked potential procedures and administered 800 500-msec flashes of highintensity blue light to each participant $(465 \mathrm{~nm} ; 9.7 \times$ $10^{14}$ photons $/ \mathrm{cm}^{2} / \mathrm{sec}$ ). No evoked response was detected at the occipital Oz location, where visual responses are expected to be the greatest, nor at any other electrode location (Supplementary Figure S1). This finding confirms a lack of image-forming light perception in either eye.

\section{Nonconscious Awareness of Light Is Present in Visually Blind Individuals}

We investigated whether the blind participants could "detect" the presence of high-intensity blue light (465 nm; $9.7 \times 10^{14}$ photons $/ \mathrm{cm}^{2} / \mathrm{sec}$ ) by performing a $2 \mathrm{AFC}$ (Zaidi et al., 2007). In addition, we varied the length of the light stimulus to examine whether the ability to detect blue light was duration dependent. In three separate sessions, participants were given 40 of either 4-, 10-, and 20-sec trials randomized for whether the first or second half of the trial included blue light exposure (i.e., 2, 5, or $10 \mathrm{sec}$ of light), with the other half consisting of darkness. Although the participants were hesitant about reporting the presence of visual stimuli that they were not conscious of, their selections were not random (Figure 1).

Responses from Participant 1 were extremely accurate (95\%) irrespective of the interval duration $\left(p<10^{-8}\right)$. The accuracy of Participant 2's responses appeared to be duration dependent with a linear increase from random

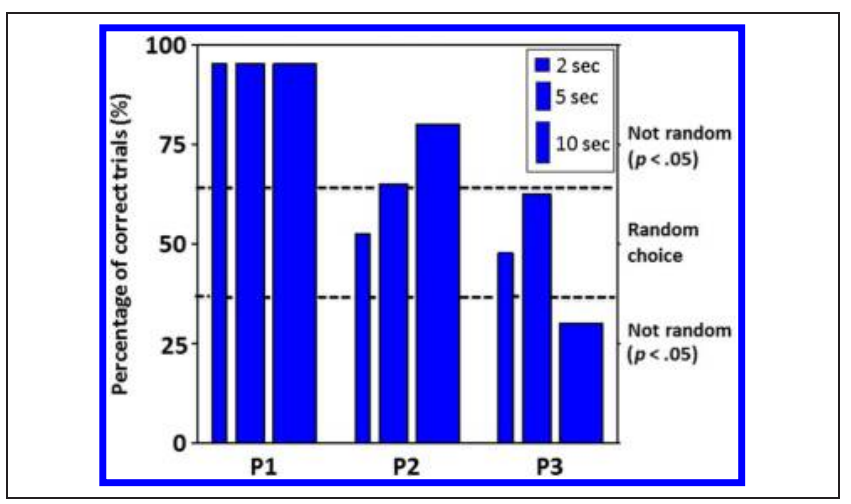

Figure 1. Results of the $2 \mathrm{AFC}$ task. Participants chose whether the light was on or off for 2,5 , or $10 \mathrm{sec}$ during the first or second half of a $4-, 10-$, or 20-sec trial, respectively. Dashed lines indicate the limit between random and nonrandom selections $(p<.05)$. $\mathrm{P} 1=$ Participant $1 ; \mathrm{P} 2=$ Participant $2 ; \mathrm{P} 3=$ Participant 3. choices for 2-sec light exposures $(p=.44), 65 \%$ accurate for 5 -sec exposures $(p=.04)$, and $80 \%$ accurate for 10-sec exposures $(p<.001)$. Participant 3 made random choices for 2 -sec light exposures $(p=.44)$ and 5 -sec exposures, although a tendency for significant detection was found for the latter exposure duration (62.5\%, $p=.08)$. When exposures were increased to $10 \mathrm{sec}$ (half of a 20 -sec epoch), his performance was poor (30\%) but significantly different from a random choice $(p=$ .008), suggesting some nonconscious awareness of the stimulation. Overall, the results indicate that at least two of the three participants could detect the presence of light during the 2AFC task, despite a complete lack of classical photoreception as measured by standard ophthalmological techniques.

\section{Two-second Blue Light Exposure Modulates EEG Activity if Administered Simultaneously to the Processing of Auditory Stimulations in Visually Blind Individuals}

We investigated whether brief high-intensity blue light exposures ( $465 \mathrm{~nm} ; 9.7 \times 10^{14}$ photons $/ \mathrm{cm}^{2} / \mathrm{sec}$ ) could modify EEG activity while participants were required to respond as fast as possible to randomly occurring auditory stimuli in a PVT, which probes the ability to maintain sustained attention (Dinges \& Powell, 1985). The goal was to investigate if a nonconsciously perceived flash of blue light could influence the EEG brain response of another (auditory) sensory modality. A similar approach is commonly used to investigate audio-visual multisensory integration in sighted individuals, in which simultaneous presentation of an auditory and a visual input produces brain integration, resulting in a greater response to simultaneous visual and auditory stimulation than the sum of the responses to each stimulation modality alone (Driver \& Noesselt, 2008).

Light and auditory stimuli were administered either alone or simultaneously, in which case, auditory stimuli were produced during the last $150 \mathrm{msec}$ of the 500-msec light exposure so that light extinction and the termination of the sound coincided. We also administered 2-sec stimuli to explore the duration-dependent impact of light, in which case, auditory stimuli were also produced during the last $150 \mathrm{msec}$ of the exposure.

No EEG response was evoked by 500 -msec or 2 -sec blue light exposures if they were administered alone (Figure 2, blue lines), confirming results of the VEP test. RTs to the PVT task were not affected by 500-msec or 2-sec light exposures ( $p>.1$; Supplementary Figure S2). Importantly, however, analysis showed that activity evoked by the processing of the auditory stimuli was reliably affected by simultaneous 2-sec light pulses in all three participants. As depicted on Figure 2 (right), at $\mathrm{Cz}$, where auditory responses are expected to be the greatest, auditory evoked responses simultaneous to light administration were significantly different not only from responses evoked 
Figure 2. Auditory evoked potentials at the $\mathrm{Cz}$ location in the presence or absence of light. EEG activity evoked by an auditory stimuli alone (green), by blue light exposure alone (blue), or by light and sound simultaneously (bimodal, red). Horizontal bars correspond to results of the permutation statistics between the bimodal trials and sum of both trials alone (probability values as on the lateral vertical color bar). The difference is significant whenever the color is not dark blue. In the 500-msec condition (left), each auditory stimulus was preceded by $350 \mathrm{msec}$ of light and terminated simultaneously with light extinction, whereas in the 2-sec condition (right), each auditory stimulus was preceded by 1850 msec of light and terminated simultaneously with light extinction. In the 2-sec condition, all three participants showed significant differences between the bimodal trials and the sum of both trials alone, whereas in the 500-msec condition, only Participant 2 presented marginal (but significant) differences (see text for details and Supplementary Figure S2 for detailed behavioral results).

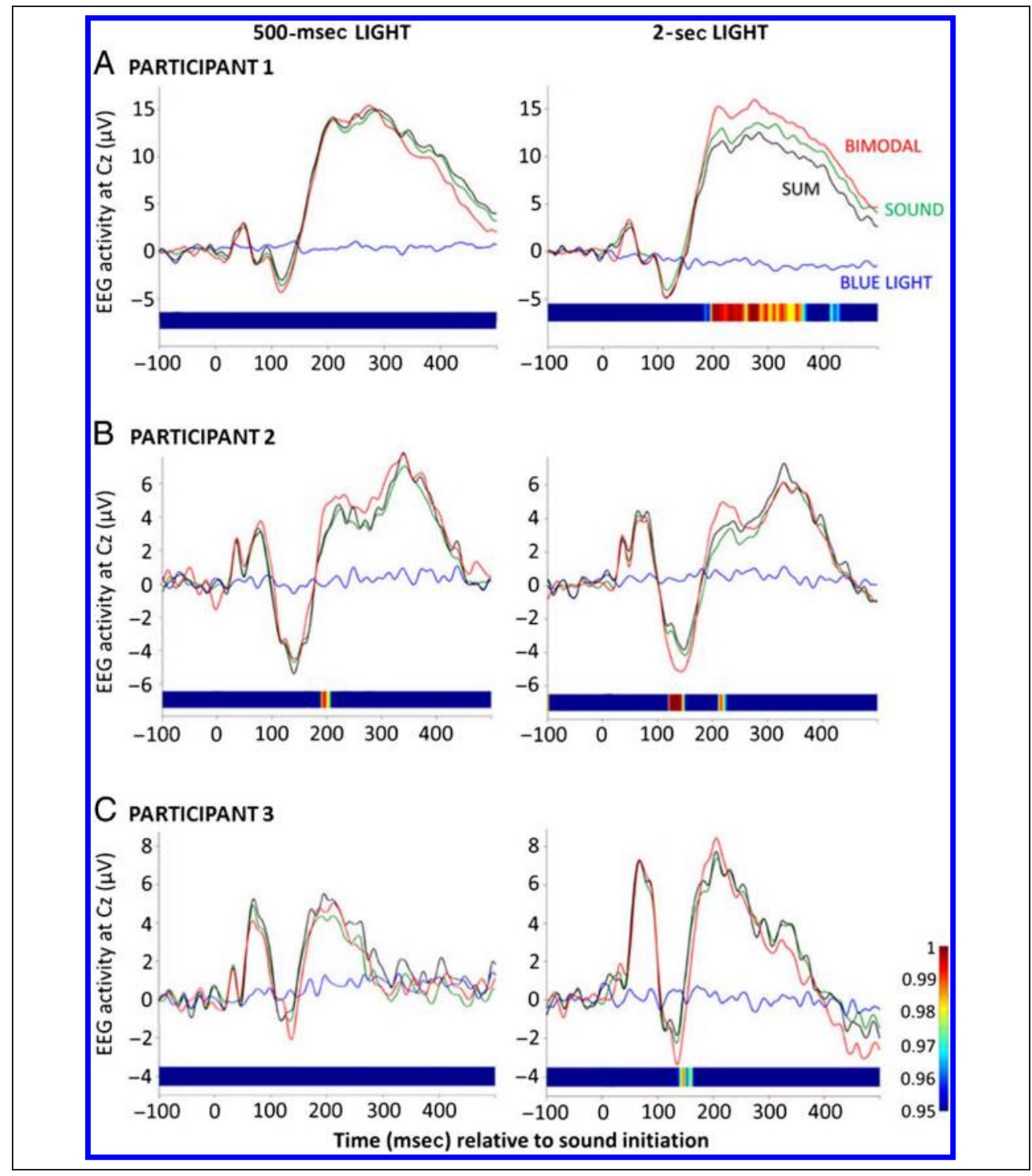

by auditory stimuli alone and by light alone but also from the sum of responses to sound and light alone (Figure 2). The amplitude of the response at Cz increased continuously between 183 and $371 \mathrm{msec}$ and between 411 and $434 \mathrm{msec}$ poststimuli for Participant 1, between 119 and $150 \mathrm{msec}$ and between 209 and $226 \mathrm{msec}$ for Participant 2, and between 137 and 163 msec for Participant 3. In contrast, the 500-msec blue light exposure had only a marginal impact on auditory evoked responses in Participant 2 (Figure 2, left), with significant amplitude increase between 189 and 206 msec.

\section{Less than a Minute of Blue Light Exposure Stimulates fMRI Brain Responses in Visually Blind Individuals}

We then employed an fMRI procedure based on previous studies in sighted individuals (Vandewalle et al., 2011;
Vandewalle, Schmidt, et al., 2007). Participants performed blocks of an auditory 2-back task alternatively in complete darkness or while exposed to high-intensity blue light for $55 \mathrm{sec}$ (480 nm; $1.95 \times 10^{14}$ photons $/ \mathrm{cm}^{2} /$ $\mathrm{sec}$ ). This task requires updating, maintaining, and comparing information in working memory, in addition to attention and auditory processing (Cohen et al., 1997). Similar to our prior findings in sighted participants (Vandewalle et al., 2011; Vandewalle, Schmidt, et al., 2007), the performance of the task was not affected by short exposures to blue light $(p>.25$; Supplementary Figure S3A) and confirms that differences in brain activation are not biased by behavioral differences.

Statistical analyses of fMRI data showed that brain activity was significantly increased under blue light exposure in numerous brain areas, as compared with complete darkness (blue $>$ dark). These areas include the ventrolateral PFC, medial PFC (MPFC), precuneus (PREC), ACC, 
and the dorso-posterior thalamus in the dorsal PULV (Figure 3, Table 2). Most of these areas are known to be involved in cognitive processes associated to the $n$-back task (Collette, Hogge, Salmon, \& Van der Linden, 2006), and we have previously reported light-induced activity modulations in many of them in sighted participants while performing the same auditory task (Vandewalle et al., 2009, 2011; Vandewalle, Gais, et al., 2007; Vandewalle, Schmidt, et al., 2007). In addition, blue light exposure increased brain activity in several regions of occipital cortex relative to darkness, including the inferior and superior occipital gyri, and the calcarine sulcus. In all these brain areas affected by the light condition, average activity estimates showed that responses to the task under blue light were superior compared with darkness (Figure 3). Individual activity estimates are also depicted in Figure 3 (right graphs) and show that activity was higher under blue light as compared with darkness in each participant but that the magnitude of the difference between the light conditions could differ substantially. Importantly, in each participant, no brain responses to the task were significantly greater in darkness compared with during blue light exposure. Furthermore, analyses examining transient brain activity associated with light onsets, to which rods and cones and the visual system are particularly responsive (Allen et al., 2011; Brown et al., 2010), yielded no significant results.

Brain responses to the task performed in darkness only exhibited a classical pattern of activations typically observed with an $n$-back task, with fronto-parietal recruitment, left lateralized in the case of a verbal task (Supplementary Figure S3B and Supplementary Table S1; Collette et al., 2006; Cohen et al., 1997). Interestingly, the significant
Figure 3. Brain areas showing significant increases in activity under blue light exposure while performing a 2-back task as compared with darkness. (Left) Statistical results $(p<.001$, uncorrected $)$ overlaid over the mean structural image of the three participants. Vertical color bars (bottom left) refer to $t$ statistic results. (Right) Activity estimates (arbitrary unit/a.u. $\pm S E M$ ) in the regions showing significant differences between blue light and darkness episodes $(p<.05$, corrected). Average activity estimates of the three participants (left) are plotted together with the individual activity estimates (right) to show the interindividual variability of the effects (see Table 2 for names of the brain regions corresponding to the letters and abbreviations and Supplementary Figure S3 and Supplementary Table S1 for detailed behavioral results, together with main brain responses to the task in darkness)

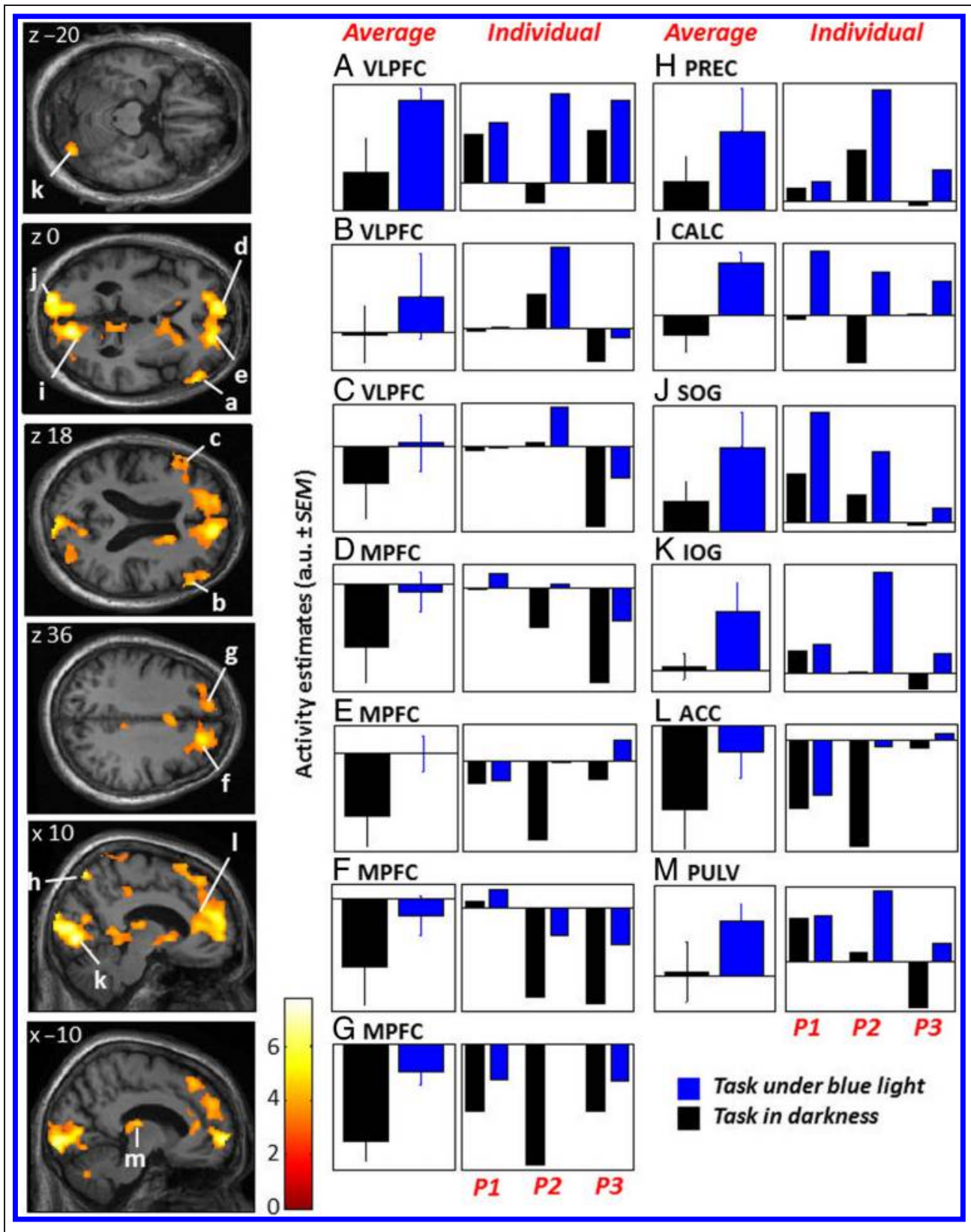


Table 2. Significant Differences between Brain Responses to an Auditory 2-Back Task Performed under Blue Light Exposure and in Darkness

\begin{tabular}{|c|c|c|c|c|}
\hline & Side & $x, y, z$ & $Z$ & $p_{\text {corrected }}{ }^{a}$ \\
\hline \multicolumn{5}{|l|}{ Blue Light $>$ Darkness } \\
\hline Ventrolateral prefrontal/frontopolar cortex & $\mathrm{R}$ & $28,52,6$ & 4.72 & .032 \\
\hline \multirow[t]{3}{*}{$\operatorname{VLPFC}[a, b, c]$} & $\mathrm{R}^{\mathrm{b}}$ & $58,38,0$ & 5.32 & .002 \\
\hline & $\mathrm{R}$ & $54,44,14$ & 5.05 & .007 \\
\hline & $\mathrm{L}$ & $-56,30,14$ & 4.61 & .049 \\
\hline \multirow[t]{7}{*}{$\operatorname{MPFC}[\mathrm{d}, \mathrm{e}, \mathrm{f}, \mathrm{g}]$} & $\mathrm{L}$ & $-12,60,-2$ & 6.88 & $<.001$ \\
\hline & $\mathrm{R}$ & $10,54,18$ & 6.19 & $<.001$ \\
\hline & $\mathrm{L}$ & $-12,50,30$ & 6.05 & $<.001$ \\
\hline & $\mathrm{R}$ & $18,40,36$ & 5.22 & .003 \\
\hline & $\mathrm{R}$ & $18,54,0$ & 4.76 & .026 \\
\hline & $\mathrm{R}$ & $16,38,50$ & 4.74 & .029 \\
\hline & $\mathrm{L}$ & $-18,56,16$ & 4.71 & .033 \\
\hline PREC [h] & $\mathrm{R}^{\mathrm{b}}$ & $8,-74,58$ & 6.59 & $<.001$ \\
\hline Calcarine sulcus [i] & $\mathrm{R}$ & $10,-82,-4$ & 7.73 & $<.001$ \\
\hline Superior occipital gyrus [j] & $\mathrm{L}^{\mathrm{b}}$ & $-18,-98,-4$ & 7.71 & $<.001$ \\
\hline Inferior occipital gyrus [k] & $\mathrm{R}^{\mathrm{b}}$ & $32,-80,-22$ & 5.03 & $<.008$ \\
\hline $\mathrm{ACC}[\mathrm{l}]$ & $\mathrm{R}$ & $10,38,10$ & 4.78 & .024 \\
\hline PULV [m] & $\mathrm{L}$ & $-10,-20,12$ & 4.64 & .044 \\
\hline
\end{tabular}

\section{Darkness > Blue Light}

No significant voxel

Letters between "[]" correspond to letters of Figure 3. $\mathrm{R}=$ right; $\mathrm{L}=$ left.

VLPFC $=$ ventrolateral prefrontal cortex; MPFC $=$ medial prefrontal cortex; PREC $=$ precuneus; PULV $=$ thalamus pulvinar.

${ }^{a} p$ Values corrected for multiple comparisons over the entire brain volume (family-wise error approach).

${ }^{\mathrm{b}}$ In these areas, an additional analyses showed that cluster size of the significant difference was slightly reduced after applying an exclusive mask $(p=$ .05 , uncorrected for multiple comparisons) for the brain response to the task blocks performed in complete darkness. All other clusters remained unaffected after mask application, supporting that blue light induces the significant recruitment of additional brain regions.

relative increases in brain responses induced by blue light did not appear to be located in areas that were significantly recruited by the task when performed in darkness. To test this assumption formally, we created a broad map of the brain areas recruited by the task in darkness in our sample, even marginally so (i.e., we displayed results of the brain responses associated to the task in darkness at a very permissive statistical threshold of $p<.05$, uncorrected for multiple comparisons), and used it to mask out the brain areas affected by blue light exposure. This procedure revealed that only four clusters found to be significant when comparing directly blue light and darkness presented a marginal overlap with the brain areas at least slightly engaged in the task in complete darkness (see footnote b in Table 2), whereas all other significant clusters showed no overlap. These results indicate that not only did blue light significantly increase brain responses when directly com- pared with darkness, but it did so mainly in areas not significantly engaged in the task during darkness.

Activity estimates showed that, in many of these areas, the effect of blue light administration, compared with darkness, was because of a reduction in the deactivation observed in the darkness condition (Figure 3C-G and L). Most of these areas, particularly the ACC and MPFC, are part of the so-called "default mode network," which constitutes a set of coupled areas that are generally disengaged relative to quiet passive wakefulness to perform an active process (Buckner, Andrews-Hanna, \& Schacter, 2008; Raichle et al., 2001). We therefore tested whether the significant differential effect of blue light versus darkness was located in areas showing significant deactivation, or disengagement when the task was performed in darkness. Deactivations in response to the task while in darkness are displayed in red on Figure 4 and correspond to 
a typical map of the default mode network (Buckner et al., 2008; Raichle et al., 2001), including areas of the MPFC; anterior, posterior, and retrosplenial cingulate; and inferior parietal cortex (see Supplementary Table S1 for a detailed list of brain regions). Widespread overlaps between these deactivations and locations of the significant impacts of blue light as compared with darkness were observed within the ACC and MPFC, demonstrating that blue light prevented the deactivations observed in complete darkness in these parts of the brain (Figure 4).

\section{DISCUSSION}

These results show that, in the absence of conscious sight, when non-image-forming photoreception is retained, light is able to modulate activity in the PULV, PREC, ACC, occipital cortex as well as in the PFC, includ-

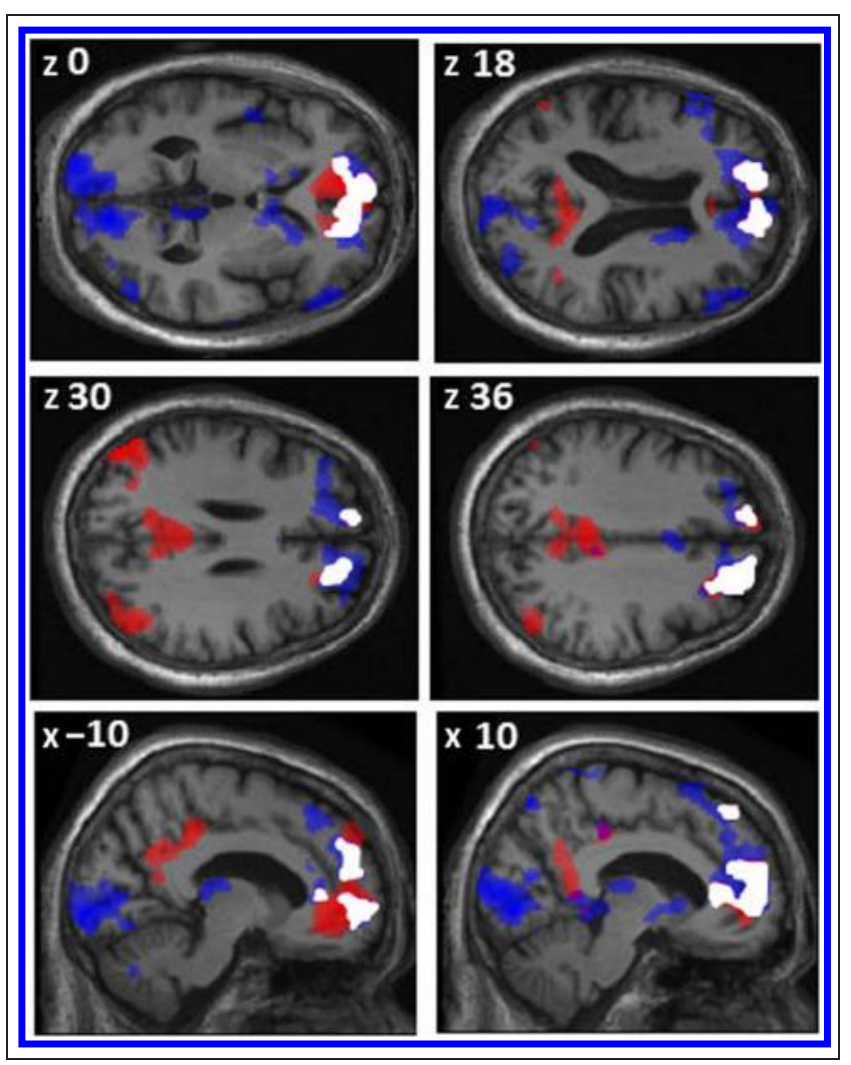

Figure 4. Blue light exposure prevents deactivation within medial prefrontal areas of the default mode network during the performance of an auditory 2-back task. Significant deactivations, as compared with baseline, in response to 2-back task during darkness are displayed in red, whereas significant differences between blocks of task performed under blue light and in complete darkness are in blue (blue light > darkness, corresponding to the results displayed in Figure 3). Overlays are displayed in white and indicate that a significant relative increase in brain response induced by blue light is located in areas showing a significant deactivation in darkness (see Supplementary Table S1 for a complete list of significant deactivations in responses to the task in darkness) ing in medial prefrontal areas of the default mode network. The results also indicate that, in our sample, administration of as little as 2 sec of high-intensity blue light is able to modulate brain activity, as shown by modification of auditory evoked EEG responses during a sustained attention task, but only when the brain is actively processing the acoustic stimulations. The data further confirm and extend the finding that rudimentary "nonconscious awareness" of light can occur in the absence of conscious vision if nonimage-forming photoreception is preserved.

A first conclusion is that the impact of light on auditory cognitive brain responses previously reported in sighted participants (Vandewalle et al., 2009, 2010, 2011) involved non-image-forming photoreception and cannot be attributed solely to conscious vision. Given the limited sample of three participants (although they represent a third of the population of such individuals identified to date), our EEG and fMRI results have to be considered as a "proof of concept" rather than a fine-grained identification of the brain mechanisms involved in the non-image-forming impact of light on cognition. Interindividual variability is reflected in the fMRI and EEG results, but also in the forced-choice task. The fact that we obtained significant results in so few cases, however, is remarkable because the onset and etiology of blindness differed substantially between participants. It is even more remarkable when considering the reduction in retinal illumination because of age-related decrease in pupil size and increase in lens yellowing, which in combination may lead, on average, to a $0.6-\log$ unit reduction in retinal illumination in a 60to 70-year-old (Kessel, Lundeman, Herbst, Andersen, \& Larsen, 2010).

Participants had no conscious light perception and no visually evoked EEG responses to 800 flashes of light, and ophthalmological examinations found no indication of residual rod or cone function. Although we cannot physically confirm the absence of functional rod and cone photoreceptors in these blind patients, the results of the EEG, fMRI, and light "nonconscious awareness" responses to blue light are consistent with the hypothesis that ipRGCs are the primary photoreceptors mediating these effects. At the irradiance we employed, the ipRGC intrinsic response, which is typically sluggish in the absence of functional rods and cones, can be detected in rodents in about a second, and this is the case even when assuming a putative 1.2-log unit age-related reduction in retinal illumination (i.e., doubled compared with normal aging; Lall et al., 2010; Dacey et al., 2005; Berson, Dunn, \& Takao, 2002). The temporal dynamics of the melanopsin response may also explain why only $2 \mathrm{sec}$ but not $500 \mathrm{msec}$ of light reliably modulated auditory-evoked EEG responses. Melanopsin's primary involvement is further supported by our recent demonstration that the dynamics, wavelength sensitivity, and sensitivity threshold of the pupillary light reflex in Participant 2 of this study matched mice lacking functional rods and cones (Gooley et al., 2012; Lucas, Douglas, \& Foster, 2001). 
Our data constitute the first direct indication that nonimage-forming photoreception alone can mediate lightinduced modulation of fMRI and EEG brain activity while performing a cognitive task and are the clearest evidence that these effects can be mediated through ipRGCs. Future studies will be able to capitalize on these initial results to isolate and quantify the contribution of nonimage-forming photoreception and ipRGCs, using additional wavelengths and different light levels in blind and sighted control individuals.

Previous studies in sighted individuals (Lockley et al., 2006; Cajochen, Zeitzer, Czeisler, \& Dijk, 2000) and a study in which Participant 2 took part (Zaidi et al., 2007) reported light-induced improvement in alertness, objectively measured by modifications in the power spectrum of waking EEG. These improvements were observed using exposures lasting several hours while participants were quietly awake, suggesting that the non-imageforming impact of light on cognitive brain function requires prolonged exposures. Similarly, significant changes in cognitive performance in sighted participants have only been reported after $30 \mathrm{~min}$ of illumination (Cajochen et al., 2011; Chellappa, Steiner, et al., 2011; Lockley et al., 2006). In this study, and consistent with previous fMRI studies in sighted participants (Vandewalle et al., 2010, 2011; Vandewalle, Schmidt, et al., 2007), changes in brain responses were observed using only 55-sec exposure. In addition, modifications of the EEG were detected with as little as $2 \mathrm{sec}$ of exposure to light and if the brain was simultaneously processing auditory information. Our data therefore demonstrate that the non-image-forming impact of light can affect cortical activity within a few seconds if brain processing is actively engaged. This finding constitutes the first indication that it is crucial to differentiate the effects of light while at rest or engaged in a cognitive process when characterizing the dynamics of the non-image-forming influence of light on brain function. In addition, our findings suggest that the bimodal PVT protocol, together with pupillary constriction test (when applicable; Gooley et al., 2012), could constitute a simple means to assess whether non-image-forming photoreception is retained in a totally blind individual, for example, before elective enucleation.

Most brain areas that showed a light-induced increase in activation in fMRI were not recruited by the task at all when it was performed in complete darkness, supporting a general increase of brain responsiveness leading to the significant recruitment of supplementary brain areas. Data previously acquired in sighted participants and in Participant 2 of this study showed that RTs in an auditory PVT or in executive tasks are improved under prolonged nighttime blue or blue-enriched light exposure (Cajochen et al., 2011; Chellappa, Steiner, et al., 2011; Zaidi et al., 2007; Lockley et al., 2006). It is therefore plausible that the recruitment of supplemental brain areas under blue light exposure we report here precedes and causes significant behavioral changes, such as RT improvements.
In addition, light-induced modulations were found in higher-order ventrolateral prefrontal areas, considered to be at the top of the hierarchy of cognitive control (Koechlin \& Hyafil, 2007). Light can therefore allow the engagement not only in supplemental areas but also in areas known to be involved in "higher level" processes. This finding clearly demonstrates that the non-image-forming photic input pathway is a strong regulator of human cognition.

Our observation that the influence of light on EEG signal could only be detected when a sound was administered supports the notion that the stimulant impact of light is initiated in subcortical structures (to which the EEG is rather insensitive) and can modulate cortical activity when it is engaged (Vandewalle et al., 2009). Targets of ipRGCs are primarily subcortical including the suprachiasmatic nucleus, ventrolateral preoptical areas, and lateral hypothalamus, which are all involved in circadian and sleep-wake regulation (Schmidt, Chen, \& Hattar, 2011). These hypothalamic structures are intimately connected with several nuclei of the brainstem ascending arousal system, such as the locus coeruleus and dorsal raphe, which regulate cognition and broadly project to the cortex (Saper, Fuller, Pedersen, Lu, \& Scammell, 2010). Light could therefore have a widespread impact on brain activation via the hypothalamus and brainstem, in which a non-image-forming effect of light has previously been suggested in sighted participants (Vandewalle et al., 2010; Vandewalle, Schmidt, et al., 2007; Perrin et al., 2004). In addition, the pulvinar, which receives indirect retinal light information through the superior colliculus (Danckert \& Rossetti, 2005) and has been shown repeatedly to be sensitive to light in studies of sighted individuals performing auditory task (Vandewalle et al., 2006, 2009, 2011; Vandewalle, Gais, et al., 2007; Vandewalle, Schmidt, et al., 2007), is also significantly affected by light in the blind participants. This finding further supports a primary role of the pulvinar in mediating the broad non-image-forming impact of light on cognition at the subcortical level, possibly through the facilitation of thalamo-cortical information flow. IpRGC output could also directly reach the occipital cortex, as previously shown in rodents (Brown et al., 2010). The occipital cortex is known, however, to be devoted to auditory processes in blind individuals because of brain plasticity phenomenon (Collignon et al., 2011) and was indeed at least slightly engaged in the task in darkness in this study (see Supplementary Figure S3B and Supplementary Table S1). It therefore remains unclear whether the significant recruitment of occipital areas during light exposure is related to the task or to direct ipRGC light information reaching these areas or both.

Given that light had an impact on another (auditory) modality, the effects we describe might be associated with multisensory integration. The time window of the modulation of the auditory evoked potential by light observed in this study (between 120 and $180 \mathrm{msec}$ ) appears congruent with several studies reporting multisensory 
modulations in higher-order regions (Driver \& Noesselt, 2008). The contribution of non-image-forming photoreception to multisensory integration is speculative and remains to be thoughtfully investigated, but our data show that the non-image-forming impact of light contributes to a multisensory interplay between exposure to light and auditory processing, likely involving ipRGCs. A site of such interplay could lie within the pulvinar, through which information can transit between areas mainly devoted to different sensory modalities (Cappe, Rouiller, \& Barone, 2009). Multisensory interplay could also take place in the superior colliculus, which is one of the most documented structures involved in multisensory integration (Stein \& Stanford, 2008) and receives direct inputs from ipRGCs (Schmidt et al., 2011; Hatori \& Panda, 2010).

The neurobiological substrate of nonconscious awareness to light remains unclear. Light awareness could be caused by the increased brain responsiveness induced by light exposure mainly through subcortical structure, which could be "sensed" by the participants but remains difficult to describe. Multisensory interplays within the pulvinar or superior colliculus could also be involved. Alternatively, direct ipRGC projections to the LGN have been demonstrated in rodents and nonhuman primates (Schmidt et al., 2011; Hatori \& Panda, 2010; Dacey et al., 2005) and could also mediate sensitivity to the presence of light. Recent data suggest that, in rodents and, possibly, in sighted humans, these ipRGC projections contribute to melanopsin-based brightness detection (Brown et al., 2012), a phenomenon that could contribute to nonconscious light awareness in our visually blind participants. Our fMRI protocol did not reveal significant light-induced activity modulations in the superior colliculus or LGN, however.

Remarkably, deactivations within the MPFC and anterior cingulate, which are part of the brain default mode network (Buckner et al., 2008; Raichle et al., 2001), were prevented when blue light was administered. This observation indicates that, in our participants, light prevented the relative disengagement of the MPFC and anterior cingulate that was normally observed when initiating a block of 2-back task in darkness. Although the role of the default mode network is still under intense investigation, two main hypotheses stand out. One postulates that the network serves internal mentation, whereas the other proposes that its role is to maintain minimal resource for the monitoring of external environment when the brain is not engaged in an active process (Buckner et al., 2008). In the latter perspective, our results raise the intriguing possibility that, during the performance of a cognitive task, light would trigger the recruitment of supplemental areas in part through the maintenance of activity within the default mode network, so that the known impact of light on sustained attention (Chellappa, Gordijn, et al., 2011; Zaidi et al., 2007; Lockley et al., 2006) would also allow the maintenance of attentional resources to monitor the environment. This theory may underlie how performance during a PVT or other executive task is improved by light exposure (Cajochen et al., 2011; Chellappa, Steiner, et al., 2011; Zaidi et al., 2007; Lockley et al., 2006).

The default mode network stands as the most robust brain functional network, and its organization appears to be similar in blind and sighted individuals (Buckner et al., 2008; Burton, Snyder, \& Raichle, 2004). The functional significance of the impact of light in some of its areas remains to be established, but the default mode network constitutes one of the fundamental organization mode of the brain (Buckner et al., 2008). To our knowledge, our results constitute the first indication that non-image-forming signaling, likely through ipRGCs, can rapidly affect basic cerebral organization, so that it could potentially participate in the regulation of numerous aspects of human brain function, in addition to the rapid recruitment of supplemental brain areas to perform an ongoing cognitive process.

\section{Acknowledgments}

We thank Amélie Apinis-Deshaies, Hélène Blais, Nathalie Bouloute, Annick Cartier, André Cyr, Stéphane Denis, Mathieu Desrosiers, Sonia Frenette, Simon Girard, Philippe GrenierVallée, Carollyn Hurst, Marjolaine Lafortune, Frédéric Lesage, Pierre Maquet, Nicolas Ouakli, Caroline Reinhardt, Manon Robert, and Zoran Sekerovic for their invaluable help. We also thank Dr. Joseph F. Rizzo III, Dr. Eliot L. Berson, and Dr. Keith H. Chiappa for performing initial neuroophthalmology examinations of the patients and Dr. Elizabeth B. Klerman for medical supervision of the melatonin suppression studies conducted in Boston. This study was supported by the Réseau Vision du Québec and Réseau de Bioimagerie du Québec (RBiQ) and the Fonds de Recherche en Santé du Québec (FRSQ). G. V. completed the analyses while being supported by the Belgian FNRS. Initial screening and melatonin suppression confirmation was supported by the National Institute of Neurological Disorders and Stroke (R01NS40982 to C. A. C. and S. W. L.); The Wellcome Trust, United Kingdom (060018/B/99/Z to S. W. L.); and the National Center for Research Resources through the Harvard Clinical and Translational Science Center at Brigham and Women's Hospital (M01RR02635 and UL1RR025758). J. T. H. was supported in part by a Cephalon Clinical Fellowship in Circadian Medicine and a National Heart, Lung and Blood Institute fellowship in Sleep, Circadian and Respiratory Neurobiology (T32 HL079010), Division of Sleep Medicine, Harvard Medical School.

Reprint requests should be sent to Gilles Vandewalle, Centre de Recherche de l'Institut Universitaire de Gériatrie de Montréal, 4565 Queen Mary, Montréal, Québec, H3W 1W5 Canada, or via e-mail: gilles.vandewalle@umontreal.ca.

\section{REFERENCES}

Allen, A. E., Brown, T. M., \& Lucas, R. J. (2011). A distinct contribution of short-wavelength-sensitive cones to lightevoked activity in the mouse pretectal olivary nucleus. Journal of Neuroscience, 31, 16833-16843.

Beck, A. T., Epstein, N., Brown, G., \& Steer, R. A. (1988). An inventory for measuring clinical anxiety: Psychometric properties. Journal of Consulting and Clinical Psychology. 56, 893-897. 
Berson, D. M., Dunn, F. A., \& Takao, M. (2002). Phototransduction by retinal ganglion cells that set the circadian clock. Science 295, 1070-1073.

Brown, T. M., Gias, C., Hatori, M., Keding, S. R., Semo, M., Coffey, P. J., et al. (2010). Melanopsin contributions to irradiance coding in the thalamo-cortical visual system. PLoS Biology, 8, e1000558.

Brown, T. M., Tsujimura, S. I., Allen, A. E., Wynne, J., Bedford, R., Vickery, G., et al. (2012). Melanopsin-based brightness discrimination in mice and humans. Current Biology, 22, 1134-1141.

Buckner, R. L., Andrews-Hanna, J. R., \& Schacter, D. L. (2008). The brain's default network: Anatomy, function, and relevance to disease. Annals of the New York Academv of Sciences. 1124, 1-38.

Burton, H., Snyder, A. Z., \& Raichle, M. E. (2004). Default brain functionality in blind people. Proceedings of the National Academv of Sciences, U.S.A., 101, 15500-15505.

Buysse, D. J., Reynolds, C. F., III, Monk, T. H., Berman, S. R., \& Kupfer, D. J. (1989). The Pittsburgh Sleep Quality Index: A new instrument for psychiatric practice and research. Psvchiatrv Research, 28, 193-213.

Cajochen, C., Frey, S., Anders, D., Spati, J., Bues, M., Pross, A., et al. (2011). Evening exposure to a light-emitting diodes (LED)-backlit computer screen affects circadian physiology and cognitive performance. Iournal of Applied Pbusiology 110, 1432-1438.

Cajochen, C., Zeitzer, J. M., Czeisler, C. A., \& Dijk, D. J. (2000). Dose-response relationship for light intensity and ocular and electroencephalographic correlates of human alertness. Behavioural Brain Research, 115, 75-83.

Cappe, C., Rouiller, E. M., \& Barone, P. (2009). Multisensory anatomical pathways. Hearing Research, 258, 28-36.

Chellappa, S. L., Gordijn, M. C., \& Cajochen, C. (2011). Can light make us bright? Effects of light on cognition and sleep. Progress in Brain Research, 190, 119-133.

Chellappa, S. L., Steiner, R., Blattner, P., Oelhafen, P., Gotz, T., \& Cajochen, C. (2011). Non-visual effects of light on melatonin, alertness and cognitive performance: Can blue-enriched light keep us alert? PLoS One, 6, e16429.

Cohen, J. D., Perlstein, W. M., Braver, T. S., Nystrom, L. E., Noll, D. C., Jonides, J., et al. (1997). Temporal dynamics of brain activation during a working memory task. Nature, 386, 604-608.

Collette, F., Hogge, M., Salmon, E., \& Van der Linden, M. (2006). Exploration of the neural substrates of executive functioning by functional neuroimaging. Neuroscience. 139, 209-221.

Collignon, O., Vandewalle, G., Voss, P., Albouy, G., Charbonneau, G., Lassonde, M., et al. (2011). Functional specialization for auditory-spatial processing in the occipital cortex of congenitally blind humans. Proceedings of the National Academv of Sciences. U.S.A., 108, 4435-4440.

Czeisler, C. A., Shanahan, T. L., Klerman, E. B., Martens, H., Brotman, D. J., Emens, J. S., et al. (1995). Suppression of melatonin secretion in some blind patients by exposure to bright light. New England Iournal of Medicine, 332, 6-11.

Dacey, D. M., Liao, H. W., Peterson, B. B., Robinson, F. R., Smith, V. C., Pokorny, J., et al. (2005). Melanopsin-expressing ganglion cells in primate retina signal colour and irradiance and project to the LGN. Nature, 433, 749-754.

Danckert, J., \& Rossetti, Y. (2005). Blindsight in action: What can the different sub-types of blindsight tell us about the control of visually guided actions? Neuroscience \& Biobehavioral Reviews, 29, 1035-1046.

Dinges, D. F., \& Powell, J. W. (1985). Microcomputer analyses of performance on a portable, simple visual RT task during sustained operations. Behavior Research Methods, Instruments, \& Computers: A Journal of the Psychonomic Society, 17, 625-655.

Dkhissi-Benyahya, O., Gronfier, C., De Vanssay, W., Flamant, F., \& Cooper, H. M. (2007). Modeling the role of midwavelength cones in circadian responses to light. Neuron, 53, 677-687.

Driver, J., \& Noesselt, T. (2008). Multisensory interplay reveals crossmodal influences on "sensory-specific" brain regions, neural responses, and judgments. Neuron, 57, 11-23.

Giard, M. H., \& Peronnet, F. (1999). Auditory-visual integration during multimodal object recognition in humans: A behavioral and electrophysiological study. Journal of Cognitive Neuroscience, 11, 473-490.

Gooley, J. J., Ho Mien, I., St Hilaire, M. A., Yeo, S. C., Chua, E. C., van Reen, E., et al. (2012). Melanopsin and rod-cone photoreceptors play different roles in mediating pupillary light responses during exposure to continuous light in humans. Iournal of Neuroscience, 32, 14242-14253.

Hannibal, J., Hindersson, P., Ostergaard, J., Georg, B., Heegaard, S., Larsen, P. J., et al. (2004). Melanopsin is expressed in PACAP-containing retinal ganglion cells of the human retinohypothalamic tract. Investigative Ophthalmology \& Visual Science, 45, 4202-4209.

Hatori, M., \& Panda, S. (2010). The emerging roles of melanopsin in behavioral adaptation to light. Trends in Molecular Medicine, 16, 435-446.

Hattar, S., Lucas, R. J., Mrosovsky, N., Thompson, S., Douglas, R. H., Hankins, M. W., et al. (2003). Melanopsin and rod-cone photoreceptive systems account for all major accessory visual functions in mice. Nature, 424, 76-81.

Horne, J. A., \& Ostberg, O. (1976). A self-assessment questionnaire to determine morningness-eveningness in human circadian rhythms. International Journal of Chronobiology, 4, 97-110.

Johns, M. W. (1991). A new method for measuring daytime sleepiness: The Epworth Sleepiness Scale. Sleep, 14, 540-545.

Kessel, L., Lundeman, J. H., Herbst, K., Andersen, T. V., \& Larsen M. (2010). Age-related changes in the transmission properties of the human lens and their relevance to circadian entrainment. Journal of Cataract \& Refractive Surgerv, 36, 308-312.

Klerman, E. B., Shanahan, T. L., Brotman, D. J., Rimmer, D. W., Emens, J. S., Rizzo, J. F., III, et al. (2002). Photic resetting of the human circadian pacemaker in the absence of conscious vision. Journal of Biological Rbvthms, 17, 548-555.

Koechlin, E., \& Hyafil, A. (2007). Anterior prefrontal function and the limits of human decision-making. Science, 318, 594-598.

Lall, G. S., Revell, V. L., Momiji, H., Al Enezi, J., Altimus, C. M., Guler, A. D., et al. (2010). Distinct contributions of rod, cone, and melanopsin photoreceptors to encoding irradiance. Neuron, 66, 417-428.

Lockley, S. W., Evans, E. E., Scheer, F. A. J. L., Brainard, G. C., Czeisler, C. A., \& Aeschbach, D. (2006). Short-wavelength sensitivity for the direct effects of light on alertness, vigilance, and the waking electroencephalogram in humans. Sleep, 29, 161-168

Lucas, R. J., Douglas, R. H., \& Foster, R. G. (2001). Characterization of an ocular photopigment capable of driving pupillary constriction in mice. Nature Neuroscience 4, 621-626.

Lucas, R. J., Freedman, M. S., Muñoz, M., Garcia-Fernández, J. M., \& Foster, R. G. (1999). Regulation of the mammalian pineal by non-rod, non-cone, ocular photoreceptors. Science, 284, 505-507.

Molholm, S., Ritter, W., Murray, M. M., Javitt, D. C., Schroeder, C. E., \& Foxe, J. J. (2002). Multisensory auditory-visual interactions during early sensory processing in humans: A 
high-density electrical mapping study. Brain Research. Cognitive Brain Research, 14, 115-128.

Panda, S., Provencio, I., Tu, D. C., Pires, S. S., Rollag, M. D., Castrucci, A. M., et al. (2003). Melanopsin is required for non-image-forming photic responses in blind mice. Science 301, 525-527.

Perrin, F., Peigneux, P., Fuchs, S., Verhaeghe, S., Laureys, S., Middleton, B., et al. (2004). Nonvisual responses to light exposure in the human brain during the circadian night. Current Biology, 14, 1842-1846.

Raichle, M. E., MacLeod, A. M., Snyder, A. Z., Powers, W. J., Gusnard, D. A., \& Shulman, G. L. (2001). A default mode of brain function. Proceedings of the National Academv of Sciences, U.S.A., 98, 676-682.

Saper, C. B., Fuller, P. M., Pedersen, N. P., Lu, J., \& Scammell, T. E. (2010). Sleep state switching. Neuron, 68, 1023-1042

Schmidt, T. M., Chen, S. K., \& Hattar, S. (2011). Intrinsically photosensitive retinal ganglion cells: Many subtypes, diverse functions. Trends in Neurosciences, 34, 572-580.

Steer, R. A., Ball, R., Ranieri, W. F., \& Beck, A. T. (1997). Further evidence for the construct validity of the Beck Depression Inventory-II with psychiatric outpatients. Psychologv Reports. 80, 443-446.

Stein, B. E., \& Stanford, T. R. (2008). Multisensory integration: Current issues from the perspective of the single neuron. Nature Reviews Neuroscience, 9, 255-266.

Vandewalle, G., Archer, S. N., Wuillaume, C., Balteau, E., Degueldre, C., Luxen, A., et al. (2011). Effects of light on cognitive brain responses depend on circadian phase and sleep homeostasis. Journal of Biological Rhythms 26, 249-259.

Vandewalle, G., Balteau, E., Phillips, C., Degueldre, C., Moreau, V., Sterpenich, V., et al. (2006). Daytime light exposure dynamically enhances brain responses. Current Biology 16, 1616-1621.

Vandewalle, G., Gais, S., Schabus, M., Balteau, E., Carrier, J., Darsaud, A., et al. (2007). Wavelength-dependent modulation of brain responses to a working memory task by daytime light exposure. Cerebral Cortex, 17, 2788-2795.

Vandewalle, G., Maquet, P., \& Dijk, D. J. (2009). Light as a modulator of cognitive brain function. Trends in Cognitive Sciences, 13, 429-438.

Vandewalle, G., Schmidt, C., Albouy, G., Sterpenich, V., Darsaud, A., Rauchs, G., et al. (2007). Brain responses to violet, blue, and green monochromatic light exposures in humans: Prominent role of blue light and the brainstem. PLoS One, 2, e1247.

Vandewalle, G., Schwartz, S., Grandjean, D., Wuillaume, C., Balteau, E., Degueldre, C., et al. (2010). Spectral quality of light modulates emotional brain responses in humans. Proceedings of the National Academv of Sciences. U.S.A. 107, 19549-19554.

Zaidi, F. H., Hull, J. T., Peirson, S. N., Wulff, K., Aeschbach, D., Gooley, J. J., et al. (2007). Short-wavelength light sensitivity of circadian, pupillary, and visual awareness in humans lacking an outer retina. Current Biology, 17, 2122-2128. 